\title{
Biological activity screening of seven Plectranthus species
}

\author{
Pesquisa de actividade biológica de sete espécies de Plectranthus
}

\author{
Epole Ntungwe N. ${ }^{1}$, Joana Marçalo ${ }^{1}$, Catarina Garcia ${ }^{1}$, Catarina Reis ${ }^{2}$, Catarina Teodósio ${ }^{1}$, Carolina \\ Oliveira ${ }^{1}$, Cláudia Oliveira ${ }^{1}$, Amílcar Roberto ${ }^{1}$, Patrícia Rijo ${ }^{1,2 *}$ \\ ${ }^{1}$ CBIOS - Research Center for Biosciences \& Health Technologies, U. Lusófona de Humanidades e Tecnologias, Campo \\ Grande 376, 1749-024 Lisboa, Portugal \\ ${ }^{2}$ Instituto de Investigação do Medicamento (iMed.ULisboa), Faculdade de Farmácia, Universidade de Lisboa, 1649-003 \\ Lisboa, Portugal \\ Email: patricia.rijo@ulusofona.pt
}

\begin{abstract}
Natural products from Plectranthus spp. plants have an ethnopharmacological use, inspiring several scientific investigations. As such, this work aims to perform a biological activity screening in order to scientifically validate the use of these plants. Assays on in vitro acetylcholinesterase (AChE) inhibition, antioxidant effects, antimicrobial activity and Artemia salina lethality were performed on seven Plectranthus spp. extracts (P. swynnertonii, P. welwischii, P. woodii, P. cylindraceus, P. spicatus, P. ramosior and P. petiolaris). Acetonic extracts were obtained by sonication $(10 \% \mathrm{w} / \mathrm{v})$, where P. ramosior had the highest yield of dry extract $(13.49 \% \mathrm{w} / \mathrm{w})$. In the AChE inhibition assay, only P. cylindraceus extract decreased enzymatic activity $(30.2 \pm 3.78 \%)$. The antimicrobial activity was screened using the well diffusion method, against Gram positive and negative bacteria and yeast. P. ramosior extract showed not only an inhibition zone against $\mathrm{S}$. aureus and C. albicans (15 and $11 \mathrm{~mm}$, respectively), but also the highest scavenging activity (DPPH method, $36.4 \pm 0.04 \%$ ). On the lethality test in A. salina, P. swynnertonnii extract was the most toxic $(\mathrm{LC} 50=0.036 \mathrm{mg} / \mathrm{L})$. These preliminary results showed that P. cylindraceus, P. ramosior and P. swynnertonnii are potential bioactive extracts for further isolation and antimicrobial and cytotoxic studies.
\end{abstract}

Keywords: Plectranthus, Acetylcholinesterase, Antioxidant, Antimicrobial, Toxicity

\section{Resumo}

Os produtos naturais das espécies de plantas Plectranthus têm um uso etnofarmacológico, suscitando vários estudos científicos. Neste trabalho pretendeu-se validar cientificamente estas plantas, realizando uma pesquisa de atividades biológicas. Extratos de sete espécies de Plectranthus (P. swynnertonii, P. welwischii, P. woodii, P. cylindraceus, P. spicatus, P. ramosior e P. petiolaris) foram testados na inibição da acetilcolinesterase in vitro (AChE), atividades antioxidante e antimicrobiana, e toxicidade em Artemia salina. Os extratos acetónicos foram obtidos por ultrassons $(10 \% \mathrm{~m} / \mathrm{v})$, dos quais P. ramosior obteve o rendimento de extração mais elevado $(13,49 \%$ $\mathrm{m} / \mathrm{m}$ ). Todos os extratos foram testados, no entanto apenas o P. cylindraceus obteve inibição da atividade da AChE $(30,2 \pm 3,78 \%)$. A atividade antimicrobiana foi avaliada usando o método de difusão em poços, contra bactérias Gram-positivas, -negativas e leveduras. P. ramosior revelou não só inibição contra S. aureus e C. albicans (15 e $11 \mathrm{~mm}$, respetivamente), como também a mais elevada atividade antioxidante (método DPPH, $36,4 \pm 0,04 \%$ ). No ensaio em A. salina P. swynnertonnii foi o extrato mais tóxico (LC50 = 0,036 mg/L).

Os resultados preliminares obtidos neste trabalho, revelam a potencial biotividade dos extratos de P. cylindraceus, P. ramosior e P. swynnertonnii, sugerindo o posterior isolamento dos compostos bioactivos, e continuidade dos estudos antimicrobianos e citotóxicos.

Palavras chave: Plectranthus, Acetilcolinesterase, Antioxidante, Antimicrobiana, Toxicidade 


\section{Introduction}

Plectranthus L' Her. genus belongs to the Lamiaceae family together with other commercially important plant genera, such as, Salvia, Ocimum or Mentha, with a rich diversity of ethnobotanical uses in traditional medicine ${ }^{(1,2)}$. The scientific validation on the traditional medicine of these plants is an important issue, to search for pharmacological activities and through drug development. In fact, there has been an increasing interest on the Plectranthus genus for the investigation of its therapeutic values ${ }^{(3,4)}$. Regarding this, the herein described study evaluated several biological activities of seven Plectranthus species (P. swynnertonii S. Moore, P. welwischii Briq. Codd, $P$. woodii Gürke, $P$. cylindraceus Hochst ex Benth, P. spicatus E. Mey ex Benth, $P$. ramosior Benth. Van Jaarsv., and $P$. petiolaris E. Mey ex Benth, Druce).

According to the references of this study, scientific information on the analyzed Plectranthus spp. is scarce and with no phytochemical studies (except for P. cylindraceus). To the best of our knowledge, this is the first report comprising the evaluation of acetylcholinesterase inhibition, antioxidant and antimicrobial activities, as well as the Artemia salina lethality assay of the seven Plectranthus species.

Alzheimer's disease (AD) is the most common cause of dementia, a progressive neurodegenerative disorder associated with memory and cognition impairment, that remains until the end of the patient life ${ }^{(5)}$. Reversible inhibition of the acetylcholinesterase (AChE) enzyme is considered as one of the prime therapeutic strategies for the treatment of $\mathrm{AD}^{(6,7)}$. Eserine, tacrine, donepezil, rivastigmine and galanthamine are the only drugs currently approved for the treatment of $\mathrm{AD}$; however, these drugs are known to have severe side effects for frequent clinical use ${ }^{(8)}$. Therefore, there is an urgent need to search for new drugs. Plectranthus spp. could be an important source to find novel compounds that can simultaneously protect neurons from oxidative stress and selectively inhibit AChE.

Humans are often exposed to free radicals which are generated from normal metabolic processes in the body. Free radicals are not always harmful, however, high level of free radicals may disturb the normal cellular mechanism by inducing oxidative damage to DNA, protein and lipids which ultimately result in chromosomal instability and mutation ${ }^{(9)}$. Moreover, overproduction of free radicals may reduce the potency of the biological defense system to detoxify these radicals which result in oxidative stress. The oxidative stress might play a role in the risk of development of several metabolic

\section{Introdução}

O género Plectranthus L' Her. pertence à família Lamiaceae juntamente com outros géneros de plantas comercialmente importantes, como Salvia, Ocimum ou Mentha, que possuem uma diversidade rica de usos etnobotânicos na medicina tradicional ${ }^{(1,2)}$. A procura de atividades farmacológicas até o desenvolvimento de fármacos, através da validação científica da medicina tradicional destas plantas é um assunto importante. $\mathrm{Na}$ verdade, tem havido um aumento na pesquisa de interesses terapêuticos do género Plectranthus ${ }^{(3,4)}$. Assim, no presente estudo são descritas várias atividades biológicas de sete espécies de Plectranthus (P. swynnertonii S. Moore, $P$. welwischii Briq. Codd, $P$. woodii Gürke, P. cylindraceus Hochst ex Benth, P. spicatus E. Mey ex Benth, P. ramosior Benth. Van Jaarsv., e P. petiolaris E. Mey ex Benth, Druce).

De acordo com as referências deste estudo, as informações científicas sobre Plectranthus spp. são escassas, e sem estudos fitoquímicos (exceto para $P$. cylindraceus). Tanto quanto é do nosso conhecimento, este é o primeiro relatório que inclui a avaliação da inibição da acetilcolinesterase, atividades antioxidantes e antimicrobianas, bem como o ensaio de letalidade de Artemia salina das sete espécies de Plectranthus.

A doença de Alzheimer (AD) é a causa mais comum de demência, um distúrbio neurodegenerativo progressivo associado à perda de memória e cognição, que permanece até o final da vida do paciente ${ }^{(5)}$. A inibição reversível da enzima acetilcolinesterase (AChE) é considerada como uma das principais estratégias terapêuticas para o tratamento da $\mathrm{AD}^{(6,7)}$. Os únicos fármacos aprovados atualmente para o tratamento da $\mathrm{AD}$ são a eserina, tacrina, donepezilo, rivastigmina e galantamina. No entanto, estes fármacos são conhecidos por terem efeitos secundários graves em uso clínico frequente ${ }^{(8)}$. Assim sendo, há uma urgente necessidade de procurar novos medicamentos. As Plectranthus spp. podem ser uma fonte essencial para encontrar novos compostos, que possam simultaneamente proteger os neurónios contra o stress oxidativo e inibir seletivamente a AChE. Os seres humanos são frequentemente expostos a radicais livres que são gerados a partir de processos metabólicos normais no corpo. Os radicais livres nem sempre são prejudiciais, no entanto, o alto nível de radicais livres pode perturbar o mecanismo celular normal, induzindo danos oxidativos ao DNA, proteínas e lipídos que, em última análise, resultam em instabilidade cromossómica e mutações ${ }^{(9)}$. Além disso, a superprodução de radicais livres pode reduzir a potência do sistema de defesa biológico para desintoxicar estes radicais, re- 
diseases such as cancer, cardiovascular diseases, diabetes, obesity, neurodegenerative and ageing-related disorders ${ }^{(9,10)}$. Regarding the antioxidant properties of the Lamiaceae family and future phytochemical evaluation of active compounds, there are several related reports of Plectranthus spp. extracts that suggest these plants as an important study motif ${ }^{(3)}$.

The antimicrobials of natural origin usually present complex chemical structures, which may be important for specific interactions and recognition of potential macromolecular targets in the pathogenic microorganisms. These active natural products are, generally, secondary plant metabolites biosynthesized to fight and prevent the proliferation of plant pathogenic microorganisms ${ }^{(11)}$. Some species of the Plectranthus genus have ethnopharmacological applications, with antimicrobial activity often cited. Indeed, it is known that both the metabolites and extracts of several Plectranthus spp. have exhibited activity against Gram-positive and Gram negative bacteria and yeasts ${ }^{(12-14)}$.

Brine shrimp lethality assay is a technique commonly used to investigate the toxic potential of plant extracts (15). This is based on cellular mechanisms that may occur via necrosis, characterised by loss of membrane integrity, death of cell or apoptosis, a genetic program of controlled cell death ${ }^{(16)}$. Brine Shrimp Lethality Assay (BSLA) has been applied as an alternative bioassay technique to screen the toxicity of plant extracts, toxicity of heavy metals and metal ions, toxicity of cyanobacteria and algae, cytotoxicity of dental materials, toxicity of nanoparticles, as well as screening of marine natural products ${ }^{(17) t}$. This test is economical and utilises small amount of test material ${ }^{(18,19)}$. The present study was therefore designed to evaluate the potential toxicity (using A. salina) of the seven Plectranthus spp.

\section{Methods and Materials}

Chemicals: Acetone, dimethyl sulfoxide (DMSO) and acetylcholine iodide (Sigma). Tacrine, HEPES buffer and Ellman reagent (Fluka Chemie AG). MuellerHinton and Sabouraud dextrose Agar, vancomycin, norfloxacine, nystatin, 2,2-diphenyl-1-picrylhydrazyl (DPPH), Quercetin, Butylated hydroxytoluene (BHT), sultando no stress oxidativo. O stress oxidativo pode ter um papel crucial no risco de desenvolvimento de várias doenças metabólicas como cancro, doenças cardiovasculares, diabetes, obesidade, distúrbios neurodegenerativos e doenças associadas ao envelhecimento $(9,10)$. Vários estudos têm vindo a avaliar os extratos de Plectranthus spp. tendo em conta as suas propriedades antioxidantes e futura avaliação fitoquímica de compostos ativos, transformando estas plantas num importante motivo de estudo ${ }^{(3)}$.

Os compostos antimicrobianos de origem natural geralmente apresentam estruturas químicas complexas que podem ser importantes nas interações específicas e reconhecimento de alvos macromoleculares potenciais, nos microrganismos patogénicos. Estes produtos naturais ativos são, em geral, metabolitos secundários vegetais biosintetizados para combater e prevenir a proliferação de microrganismos patogénicos das plantas ${ }^{(11)}$ Algumas espécies do género Plectranthus têm aplicações etnofarmacológicas com atividade antimicrobiana frequentemente citada. De facto, sabe-se que tanto os metabolitos como os extratos de várias Plectranthus têm demonstrado atividade contra bactérias Gram-positivas e negativas e leveduras ${ }^{(12-14)}$.

O ensaio de letalidade em Artemia salina é uma técnica usual para avaliar o potencial tóxico de extratos vegetais ${ }^{(15)}$. Este ensaio é baseado no mecanismo celular que pode ocorrer através de necrose, caracterizada pela perda da integridade da membrana, morte da célula ou apoptose, um programa genético de morte celular controlada ${ }^{(16)}$. O ensaio de letalidade em A. salina (BSLA) tem vindo a ser aplicado como uma técnica alternativa de bioensaio para examinar a toxicidade de extratos de plantas, toxicidade de metais pesados e iões metálicos, toxicidade de cianobactérias e algas, citotoxicidade de materiais dentários, toxicidade de nanopartículas, bem como no rastreio de produtos marinhos naturais ${ }^{(17)}$. Este teste é económico e necessita apenas de uma pequena quantidade de amostra ${ }^{(18,19)}$. O presente estudo foi portanto concebido para avaliar a potencial toxicidade (utilizando o modelo de $A$. salina) das sete espécies de Plectranthus.

\section{Métodos}

Reagentes: Acetona, dimetilsulfóxido (DMSO) e iodeto de acetilcolina (Sigma). Tacrina, tampão HEPES e reagente de Ellman (Fluka Chemie AG). Agar Mueller-Hinton e Sabouraud Dextrose, vancomicina, norfloxacina, nistatina, 2,2-difenil-1-picrilhidrazilo (DPPH), quercetina, hidroxitolueno butilado (BHT), metanol, 
Methanol, JBL Artemia salt, JBL Artemia eggs (JBL GmbH and Co. KG, D-67141 Neuhofen Germany).

Equipment: Rotary evaporator (K Ika-WERKE, GMBH \$ CO.KG; D-79219 Staufen Germany), spectrophotometer U-1500 Hitachi Instruments, Inc USA., microscope (E, CETI Belgium), 24 well microplates, aquarium air pump (HI-FLO ${ }^{\mathrm{TM}}$ Single Type 4000), Thermostat Cabinet AQUA LYTIC ${ }^{\circledR}$, TLC silica gel $60 \mathrm{~F}_{254} 20 \times 20$ (Merck KGaA), Whatman, Inc., Clifton, NJ, USA and ultrasound apparatus from VWR.

\section{Plant material}

All plant materials of Plectranthus swynnertonii S. Moore, $P$. welwischii (Briq. Codd), $P$. woodii Gürke, $P$. cylindraceus Hochst, ex Benth, P. spicatus E. Mey ex Benth., P. ramosior Benth. Van Jaarsv., P. petiolaris E. Mey ex Benth, Druce were grown in the Parque Botânico da Tapada da Ajuda from cuttings provided by the Kirstenbosch National Botanical Gardens, South Africa, and were collected between 2007 and 2008, always in June and September, and voucher specimens were deposited in the Herbarium "João de Carvalho e Vasconcellos" of the "Instituto Superior de Agronomia", Lisboa (LISI), Portugal.

\section{Microorganisms}

Enterococcus faecalis ATCC 29212, Escherichia coli ATCC 25922, Pseudomonas aeruginosa ATCC 27853, Staphylococcus aureus ATCC 25923, Saccharomyces cerevisiae ATCC 2601 and Candida albicans ATCC 10231.

\section{Extract preparation}

Plant extracts were prepared by a sonication extraction method $(10 \%(\mathrm{w} / \mathrm{v}))$. This was performed by adding $30 \mathrm{~mL}$ of acetone to $3 \mathrm{~g}$ of grinded dry plant leaves three times, sonicating for 1 hour for each extraction, and filtering (Whatman $\mathrm{N}^{\mathrm{o}} 5$ paper, Inc., Clifton, NJ, USA.) ${ }^{(14)}$ after each sonication. The liquid samples were evaporated using a rotary evaporator at $40-50^{\circ} \mathrm{C}$. The extracts were prepared at the concentration of $10 \mathrm{mg} /$ $\mathrm{mL}$ in DMSO. The extraction yield $(\% \mathrm{w} / \mathrm{w})$ for each of the extracts was determined (Equation 1).
JBL Artemia salt, JBL Artemia eggs (JBL GmbH e Co. KG, D-67141 Neuhofen Alemanha).

Equipamento: Evaporador rotativo (K Ika-WERKE, GMBH \$ CO.KG; D-79219 staufen Alemanha), Espectrofotómetro U-1500 Hitachi Instruments, Inc USA., Microscópio óptico (E, CETI Bélgica), microplacas de 24 poços, Bomba de ar de aquário (HI-FLO ${ }^{\mathrm{TM}}$ Single Type 4000), Armário com termóstato AQUA LYTIC ${ }^{\circledR}$, silica gel de TLC $60 \mathrm{~F}_{254} 20 \times 20$ (Merck KGaA), Whatman, Inc., Clifton, NJ, USA e aparelho de ultrassons da VWR.

\section{Material vegetal}

Todo o material das plantas Plectranthus swynnertonii S. Moore, P. welwischii (Briq. Codd), P. woodii Gürke, P. cylindraceus Hochst, ex Benth, P. spicatus E. Mey ex Benth., P. ramosior Benth. Van Jaarsv. e P. petiolaris E. Mey ex Benth, Druce cresceu no Parque Botânico da Tapada da Ajuda, provenientes de porções de caules vindos do "Kirstenbosch National Botanical Gardens", África do Sul, e foi recolhido entre 2007 e 2008, sempre em Junho e Setembro e variados espécimes foram plantados no Herbário "João de Carvalho e Vasconcelos" do "Instituto Superior de Agronomia", Lisboa (LISI), Portugal.

\section{Microorganismos}

Enterococcus faecalis ATCC 29212, Escherichia coli ATCC 25922, Pseudomonas aeruginosa ATCC 27853, Staphylococcus aureus ATCC 25923, Saccharomyces cerevisiae ATCC 2601 e Candida albicans ATCC 10231.

\section{Preparação dos extratos}

Os extratos das plantas foram preparados utilizando um método de extração por ultrassons $(10 \%(\mathrm{~m} / \mathrm{v})$. Adicionaram-se $30 \mathrm{~mL}$ de acetona a $3 \mathrm{~g}$ de folhas trituradas de planta seca, três vezes durante 1 hora cada extração, e filtrou-se usando filtros Whatman papel $\mathrm{N}^{\circ} 5$ (Whatman, Inc., Clifton, NJ, USA.) ${ }^{(20)}$. Os extratos obtidos foram evaporados usando o evaporador rotativo a 40$50{ }^{\circ} \mathrm{C}$. O extratos foram depois colocados em Eppendorfs e dissolvidos em DMSO a uma concentração de $10 \mathrm{mg} / \mathrm{mL}$. O rendimento de extração foi determinado para cada um dos extratos (Equação 1).

$$
\begin{aligned}
& \text { Equation 1: \% Yield }(\mathrm{w} / \mathrm{w})=\frac{\text { Weight of extract }(\mathrm{g})}{\text { Weight of dried plant }(\mathrm{g})} \times 100 \\
& \text { Equação 1: } \% \text { Rendimento }(\mathrm{m} / \mathrm{m})=\frac{\text { Residuo } \sec 0(\mathrm{~g})}{\text { Planta seca }(\mathrm{g})} \times 100
\end{aligned}
$$




\section{In vitro Acetylcholinesterase inhibition}

Acetylcholinesterase enzymatic activity was evaluated in accordance with the method described by Rijo et al., ${ }^{(20)}$ using a microplate reader. All tests were carried out in triplicate with tacrine as the positive control. The acetonic extracts were tested at a concentration of 0.1 $\mu \mathrm{g} / \mathrm{mL}$, and the inhibition percentage values were calculated according to Equation 2 and $\mathbf{3}$.
Atividade da acetilcolinesterase in vitro

A atividade enzimática da acetilcolinesterase foi avaliada de acordo com o método descrito por Rijo et al., ${ }^{(20)}$ usando um leitor de microplacas (Secção 2.2).Todos os testes foram executados em triplicado usando tacrina como controlo positivo. Os extratos das plantas foram testados a uma concentração de $0,1 \mu \mathrm{g} / \mathrm{mL}$ de acordo com as equações em Equação 2 e 3.

$$
\text { Equation 2: Velocity reaction of control or inhibitor }=\frac{\text { Corrected absorbance }(\mathrm{nm})}{\text { Time }(\mathrm{min})}
$$

$$
\text { E quação 2: Velocidade da reação do controlo ou inibidor }=\frac{\text { Absorvência corrigida }(\mathrm{nm})}{\text { Tempo }(\mathrm{min})}
$$

Equation 3: Inhibition $(\%)=100-\left(\frac{100 \times \text { Velocity reaction of inhibit or }}{\text { Velocity reaction of control }}\right)$

$$
\text { Equação 3: Inibição }(\%)=100-\left(\frac{100 \times \text { Velocidade da reação do inibidor }}{\text { Velociđade da reação controlo }}\right)
$$

\section{Antimicrobial activity: Well Diffusion Method}

The antimicrobial activity of each prepared extract was evaluated against two Gram-positive bacteria (E. faecalis and $S$. aureus), two Gram-negative bacteria (E. coli and $P$. aeruginosa) and two yeasts ( $S$. cerevisiae and C. albicans). Previously prepared extracts as described previously were reconstituted in DMSO at $1 \mathrm{mg} / \mathrm{mL}$ concentration. Stock solutions of reference antibiotics (vancomycin, norfloxacin and nystatin) were also prepared at $1 \mathrm{mg} / \mathrm{mL}$ in DMSO.

In aseptic conditions, Petri dishes containing $20 \mathrm{~mL}$ of solid Mueller-Hinton, or Sabouraud Dextrose Agar culture medium for yeasts, were inoculated with $0.1 \mathrm{~mL}$ of bacterial suspension matching a $0.5 \mathrm{McF}$ arland standard solution and uniformly spread on the medium surface using a sterile swab. Wells of approximately $5 \mathrm{~mm}$ in diameter were made in the medium, using a sterile glass Pasteur pipette, and $50 \mu \mathrm{L}$ of each extract were added into the wells. A positive control of vancomycin for Gram-positive bacteria, norfloxacin for Gram-negative bacteria and nystatin for yeasts and a negative control of DMSO were used in the assay. Plates were incubated at $37^{\circ} \mathrm{C}$ for 24 hours. The antimicrobial activity was evaluated by measuring the diameter $(\mathrm{mm})$ of the inhibition zone formed around the wells and compared to controls ${ }^{(4)}$.
Atividade antimicrobiana: Método de difusão em poços

A atividade antimicrobiana de cada extrato preparado foi avaliada contra seis espécies bacterianas e leveduras obtidas de "American Type Culture Collection" (ATCC), nomeadamente Enterococcus faecalis ATCC 29212, Escherichia coli ATCC 25922, Pseudomonas aeruginosa ATCC 27853, Staphylococcus aureus ATCC 25923, Saccharomyces cerevisiae ATCC 2601 e Candida albicans ATCC 10231. O ensaio de difusão em poços foi usado para selecionar os extratos com atividade antimicrobiana.

Os extratos preparados anteriormente de acordo com o descrito na secção $\mathbf{2 . 5}$ foram reconstituídos em DMSO a uma concentração de $1 \mathrm{mg} / \mathrm{mL}$. As soluções stock dos antibióticos de referência (vancomicina, norfloxacina e nistatina) foram também preparadas a uma concentração de $1 \mathrm{mg} / \mathrm{mL}$ em DMSO.

Em condições assépticas, as caixas de Petri que continham $20 \mathrm{~mL}$ de meio de cultura de Agar Mueller-Hinton sólido e Sabouraud Dextrose (para as leveduras) foram inoculadas com $0,1 \mathrm{~mL}$ de suspensão bacteriana de acordo com a escala 0,5 da solução de McFarland e a suspensão foi uniformemente espalhada na superfície do meio usando um cotonete estéril. Foram feitos poços com aproximadamente $5 \mathrm{~mm}$ de diâmetro no meio com uma pipeta de Pasteur de vidro esterilizada, e foram co- 


\section{Determination of Antioxidant Activity (DPPH)}

The antioxidant activity of all extracts was measured by DPPH method, as described by Rijo et al. ${ }^{(20)} 10 \mu \mathrm{L}$ of each extract sample were added to a $990 \mu \mathrm{L}$ solution of DPPH $(0.002 \%$ in methanol). The mixture was incubated for 30 minutes at room temperature. The absorbance was measured at $517 \mathrm{~nm}$ against a corresponding blank and the antioxidant activity was calculated using Equation 4. locados $50 \mu \mathrm{L}$ de cada extrato nesses poços. O controlo positivo usado foi a vancomicina para bactérias Gram-positivas, a norfloxacina para bactérias Gram-negativas, a nistatina para fungos e leveduras e o controlo negativo usado foi o DMSO. As placas foram incubadas a $37^{\circ} \mathrm{C}$ durante 24 horas. A atividade antibacteriana foi avaliada por medição do diâmetro $(\mathrm{mm})$ da zona de inibição formada à volta dos poços e comparando-os com o diâmetro dos controlos observados.

\section{Determinação da atividade antioxidante (DPPH)}

Para avaliar a capacidade de recaptação de radicais do extrato, foi usado o método do DPPH ${ }^{(20)}$. Foram adicionados $10 \mu \mathrm{L}$ de extrato de planta (amostra) a $990 \mu \mathrm{L}$ de solução de DPPH $(0,002 \%$ em metanol). A mistura foi incubada durante 30 minutos à temperatura ambiente. A absorvência foi medida a $517 \mathrm{~nm}$ contra o correspondente branco e a atividade antioxidante foi calculada de acordo com a Equação 4:

$$
\begin{aligned}
& \text { Equation 4: AA }(\%)=\frac{A \text { DPPH }-A \text { sample }}{A \text { DPPH }} \times 100 \\
& \text { Equação 4: AA (\%) }=\frac{A \text { DPPH }-A \text { amostra }}{A \text { DPPH }} \times 100
\end{aligned}
$$

AA is the antioxidant activity, A DPPH is the absorption of DPPH against the blank and A sample is the absorption of the extract or control against the blank. Tests were carried out in triplicate at a sample concentration at $1 \mathrm{mg}$ of dry plant extract $/ \mathrm{mL}$. The reference standard used for this procedure was quercetin, used in the same conditions as the samples.

\section{Brine shrimp lethality bioassay}

In order to evaluate the toxicity of the different extracts, a test of lethality to Artemia salina brine shrimp was performed ${ }^{(18)}$. Concentrations of $10 \mathrm{ppm}$ of each extract were tested. The number of dead larvae was recorded after 24 hours and used to calculate the lethal concentration (\%), according to Equation 5.
Em que AA é a atividade antioxidante, A DPPH é a absorvência do DPPH contra o branco e A amostra é a absorvência do extrato ou controlo contra o branco. Os testes foram feitos em triplicado. Os extratos foram testados a uma concentração final de $10 \mu \mathrm{g} / \mathrm{mL}$.

\section{Bioensaios de letalidade em Artemia salina}

Com o objetivo de avaliar a toxicidade dos diferentes extratos foi realizado um teste de letalidade usando o modelo Artemia salina ${ }^{(18)}$. Foram testadas concentrações de 10 ppm de cada extracto. O número de larvas mortas foi registado após $24 \mathrm{~h}$ e utilizado para calcular a percentagem (\%) de concentração letal, de acordo com

\section{a Equação 5.}

Equation 5: Lethal concentration $(\%)=\frac{\text { Total nauplii }- \text { Alivenauplii }}{\text { Total nauplii }}$

Equação 5: Concentração letal $(\%)=\frac{\text { Náuplios totais }- \text { Náuplios vivos }}{\text { Náuplios totais }}$ 


\section{Results}

Extraction yield: Seven Plectranthus species were used in this study. The amount of dry extract weight of each Plectranthus species is presented in Figure 1. $P$. ramosior had the highest extraction yield (13.49\% w/w), followed by $P$. petiolaris while $P$. welwischii had the least extraction yield $(3.59 \% \mathrm{w} / \mathrm{w})$.

In vitro Acetylcholinesterase inhibition: The $\mathrm{AChE}$ percentage inhibition was evaluated in all extracts at a concentration of $1 \mathrm{mg} / \mathrm{mL}$, and once more $P$. cylindraceus $(30.2 \pm 3.78 \%$ inhibition) extract was the most active, as is shown in Figure 2.
Os extratos ativos (acima dos $40 \%$ ) foram novamente testados às concentrações de $0.1,0.5$ e 1 ppm. O número de larvas mortas foi registado e usado para calcular a Concentração Letal, 50\% $\left(\mathrm{LC}_{50}\right)$ que foi determinada após 24 horas e a contagem foi feita usando um microscópio. Os valores de $\mathrm{LC}_{50}$ menores ou iguais a $1 \mathrm{ppm}$ foram considerados ativos.

\section{Resultados}

Rendimento: Sete espécies do género Plectranthus foram usadas neste estudo. A quantidade dos extratos secos de cada uma das espécies de Plectranthus está apresentada na Figura 1. P. ramosior teve a maior rendimento de extração $(13,49 \% \mathrm{~m} / \mathrm{m})$, seguido pelo $P$. petiolaris enquanto que o $P$. welwichii obteve o resultado mais baixo $(3,59 \% \mathrm{~m} / \mathrm{m})$.

Inibição da acetilcolinesterase in vitro: A inibição de AChE foi avaliada em todos os extratos $(1 \mathrm{mg} / \mathrm{mL})$, e uma vez mais o extrato $P$. cylindraceus $(30,2 \pm 3,78 \%$ inibição) foi o mais ativo, como representado na Figura 2.

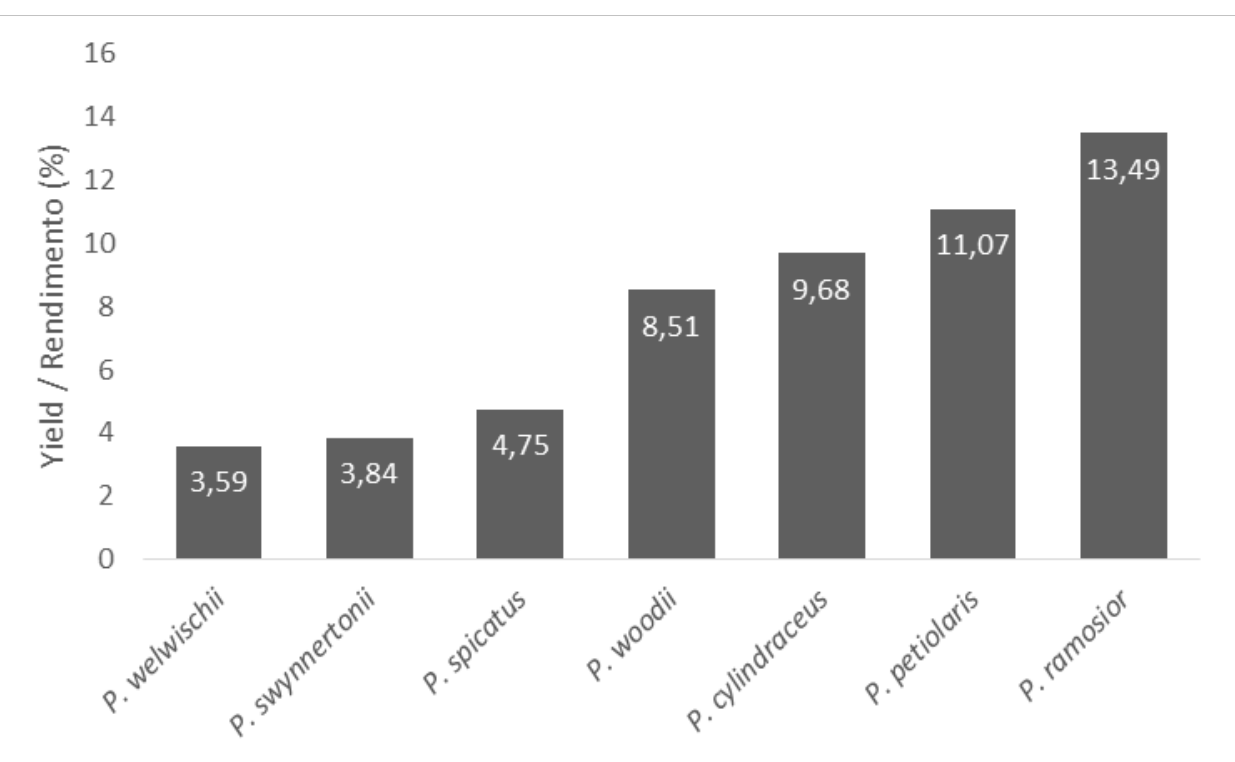

Figure 1 / Figura 1- Percentage yield (dry weight \% w/w) of seven Plectranthus species / Rendimento (resíduo seco \% m/m) das sete espécies de Plectranthus. 


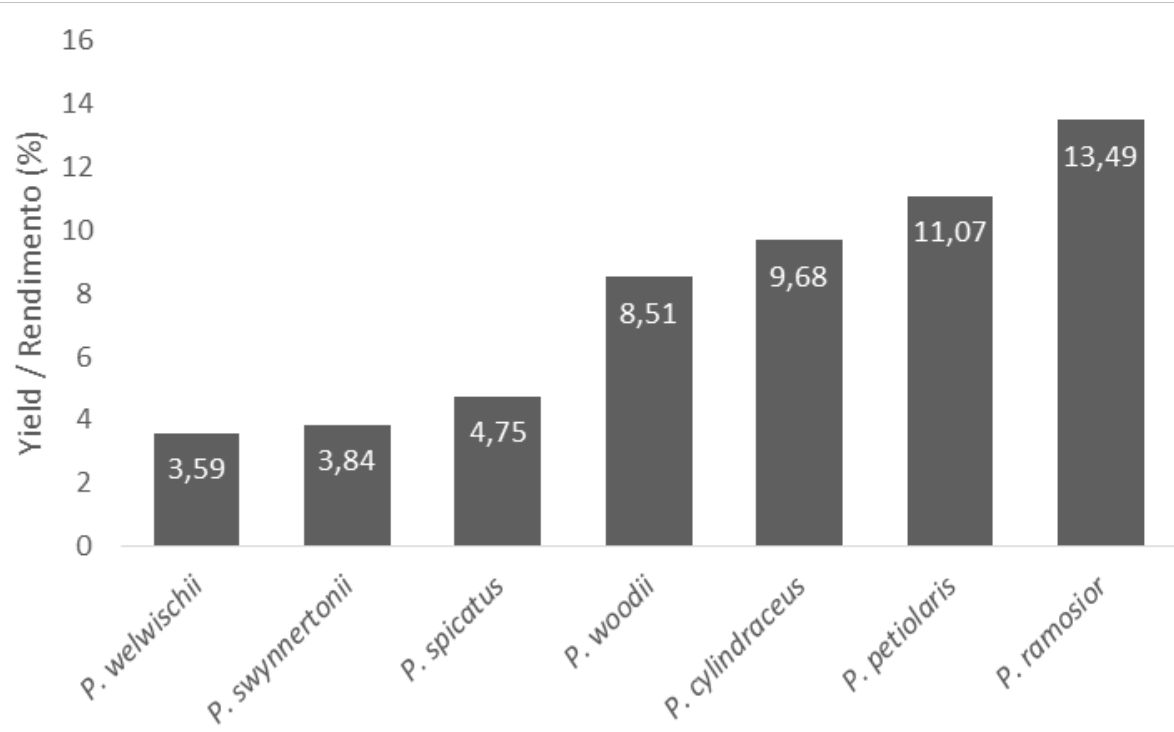

Figure 2 / Figura 2 - Acetylcholinesterase (AChE) percentage inhibition for seven Plectranthus species at $1 \mathrm{mg} / \mathrm{mL} /$ Percentagem de inibição da acetilcolinesterase (AChE) para as sete espécies de Plectranthus a $1 \mathrm{mg} / \mathrm{mL}$.

Antimicrobial activity: The antimicrobial activity of the extracts was evaluated by the well diffusion method. Considering all extracts, the acetonic extract of $P$. ramosior obtained was the more bioactive. $P$. ramosior was active against both $S$. aureus and $C$. albicans (Table 1). None of the extracts showed significant antibacterial activity against Gram-negative bacteria with inhibition zones identical to that obtained with the negative control DMSO (5 mm).

\section{Antioxidant activity (DPPH assay)}

The antioxidant activity of the Plectranthus spp. acetonic extracts in study was evaluated using the DPPH method. The antioxidant activity of the extracts was qualitatively determined using the DPPH radical assay in TLC ${ }^{(14)}$ and all the extracts studied yielded positive results. The extracts were then quantified, and P. ramosior showed the highest scavenging activity (36.24\%) and P. spicatus the least (10.57\%) (Figure 3).

\section{Brine shrimp lethality bioassay}

In this study, Artemia salina model was preliminary used to assess the toxicity of all extracts ${ }^{(19)}$. Only three of the extracts were toxic to A. salina, as shown in Figure 4 . The active extracts (LC above $40 \%$ ) were further determined at concentrations of $0.1,0.5$ and $1 \mathrm{ppm}$. The number of dead larvae was recorded and used to calculate the Lethal Concentration, 50\% $\left(\mathrm{LC}_{50}\right)$, after $24 \mathrm{~h} . \mathrm{LC}_{50}$ values less than or equal to $1 \mathrm{ppm}$ were considered active (Table 2).
Atividade antimicrobiana: A atividade antimicrobiana dos extratos foi avaliada pelo método de difusão em poços. Considerando todos os extratos, o extrato de $P$. ramosior obtido foi o mais bioativo. P. ramosior foi ativo contra $S$. aureus e $C$. albicans (Tabela 1). Nenhum dos extratos mostrou atividade antibacteriana significativa contra bactérias Gram-negativas com zonas de inibição idênticas às obtidas com o controlo negativo DMSO (5 mm).

\section{Atividade antioxidante:}

A atividade antioxidante dos extratos de Plectranthus foi avaliada pelo método de DPPH. A atividade antioxidante dos extratos foi qualitativamente determinada utilizando o ensaio de radical DPPH em TLC ${ }^{(12)}$ e todos os extratos estudados produziram resultados positivos. Os extratos foram então quantificados e extrato de $P$. ramosior apresentou a maior atividade antioxidante $(36.24 \%)$ enquanto que o $P$. spicatus obteve o resultado menor (10.57\%) (Figura 3).

\section{Bioensaio da letalidade em Artemia salina:}

O modelo de A. salina foi utilizado preliminarmente para avaliar a toxicidade de todos os extratos neste estudo ${ }^{(19)}$. Apenas três dos extratos foram considerados tóxicos para $A$. salina, como mostrado na Figura 4. Os extratos ativos (LC acima de $40 \%$ ) foram adicionalmente determinados a concentrações de 0,$1 ; 0,5$ e 1 ppm. O número de náuplios mortos foi registado e utilizado para calcular a Concentração Letal, 50\% $\left(\mathrm{LC}_{50}\right)$, após 24 horas. Valores de $\mathrm{LC}_{50}$ inferiores ou iguais a $1 \mathrm{ppm}$ foram considerados ativos (Tabela 2). 
Table 1/ Tabela 1 - Antimicrobial activity of the acetonic extracts obtained by the well diffusion method $(\mathrm{mm})$ / Atividade antimicrobiana dos extratos acetónicos, obtida pelo método de difusão em poços $(\mathrm{mm})$.

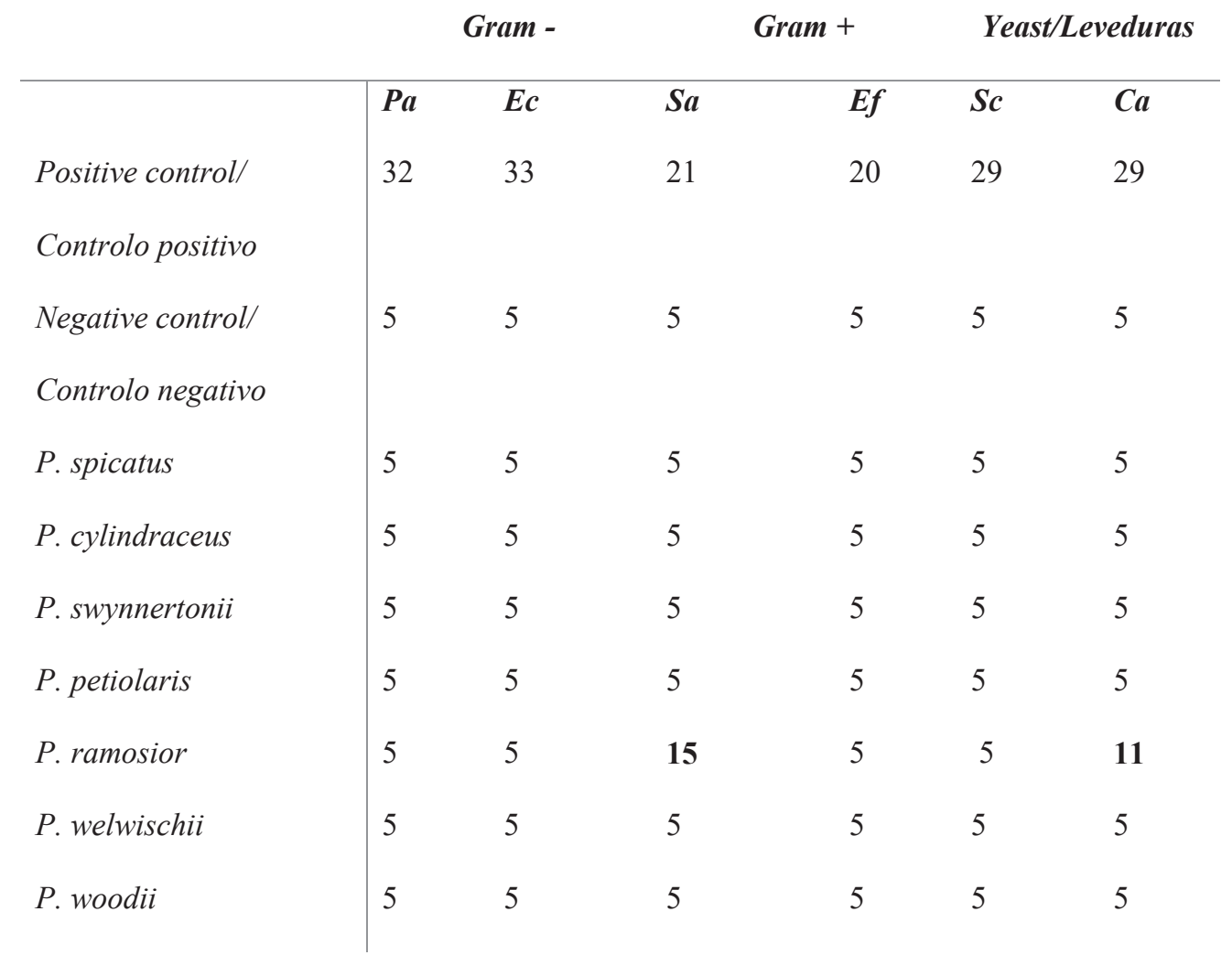

Pa - Pseudomonas aeruginosa; Ec - Escherichia coli; Sa - Staphylococcus aureus; Ef Enterococcus faecalis; Sc - Saccharomyces cerevisiae; Ca-Candida albicans.

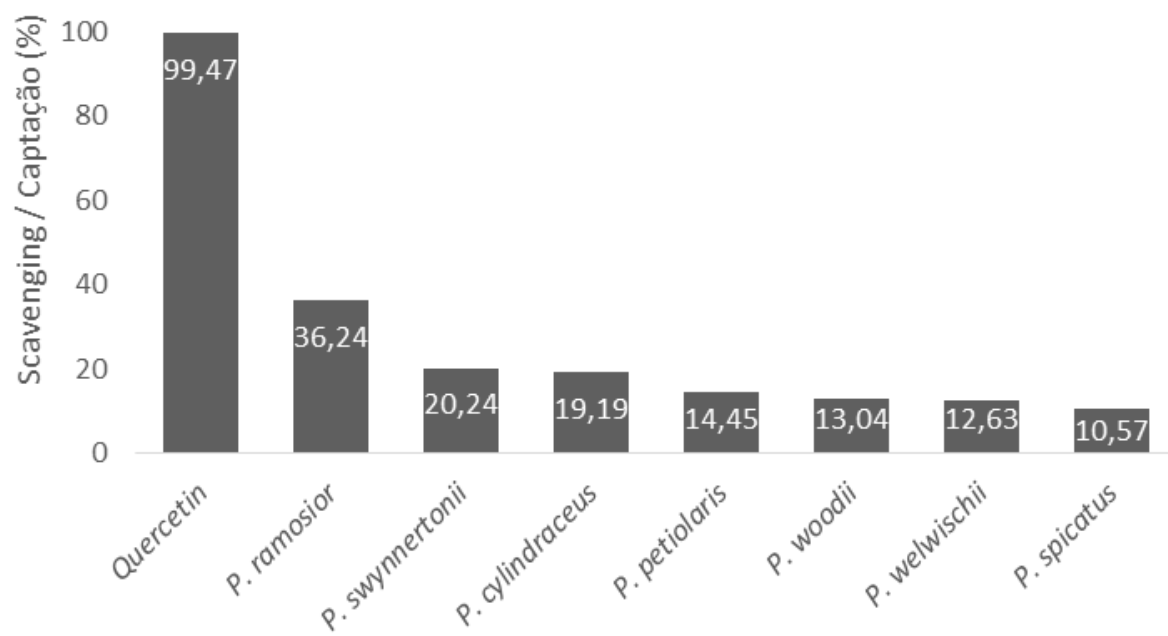

Figure 3 / Figura 3 - Antioxidant activity of extracts under study tested at a concentration of $10 \mu \mathrm{g} / \mathrm{mL} /$ Atividade antioxidante dos extratos em estudo tesados à concentração de $10 \mu \mathrm{g} / \mathrm{mL}$. 


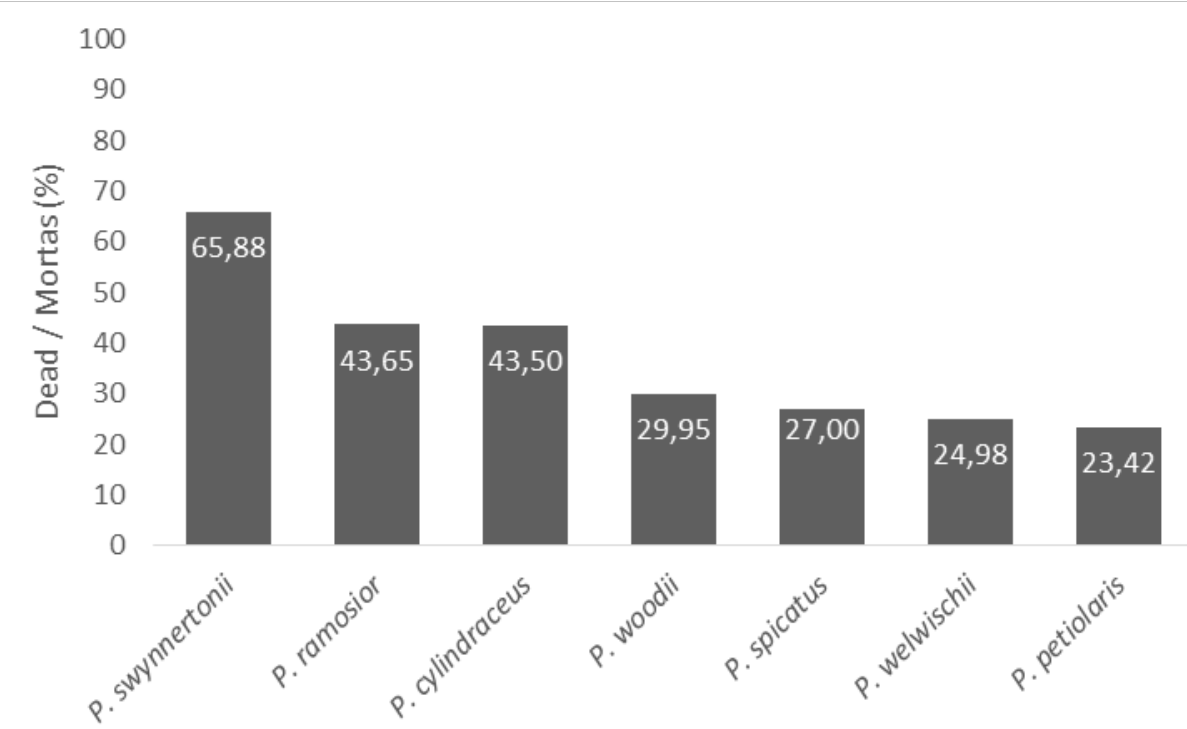

Figure 4 / Figura 4 - Screening of seven Plectranthus spp. extracts for toxicity at a concentration of $10 \mathrm{ppm}$ using the Artemia salina test /

Pesquisa de atividade de extratos de sete espécies de Plectranthus para toxicidade à concentração de 10 ppm usando o teste de Artemia salina.

Table 2 / Tabela 2 - LC50 values (mg/L) for the most active extracts using the Artemia salina test /

Valores de LC50 (mg/L) para os extratos mais ativos usando o teste de Artemia salina.

\begin{tabular}{|c|c|}
\hline Extracts/Extratos & $L C_{50}(\mathrm{mg} / \mathrm{L})$ \\
\hline P. ramosior & 0,88 \\
\hline P. cylindraceus & 0,504 \\
\hline P. swynnertonnii & 0,036 \\
\hline
\end{tabular}




\section{Discussion}

In the present study, the acetylcholinesterase inhibition, antioxidant and antimicrobial activities and Artemia salina toxicity of seven Plectranthus spp. (P. swynnertonii, $P$. welwischii, $P$. woodii, $P$. cylindraceus, $P$. spicatus, $P$. ramosior and $P$. petiolaris) were investigated. The acetonic extracts of the seven Plectranthus species were prepared using an ultrasound-assisted extraction method. Yields of extraction were determined for all prepared extracts, and the $P$. ramosior acetonic extract revealed higher yields (Figure 1). Thus, the enhanced yield extraction of $P$. ramosior is related to the greater extent of cell rupture of the plant material. This is probably due to the ultrasound extraction that causes the migration of compounds from the plant material, providing an enriched extract compared to the other extracts in study ${ }^{(20)}$.

Regarding in vitro AChE inhibition, P. cylindraceus acetonic extract showed the highest inhibition (Figure 2), and may have the highest content in rosmarinic acid, since a correlation between rosmarinic acid content and AChE inhibition capacity has been reported previously for four other Plectranthus species (P. ecklonii, P. fruticosus, P. lanuginosus and P. verticillatus) ${ }^{(20)}$. The AChE inhibition of these Plectranthus spp. under study was relatively low comparing to some of the synthetic medicines used, e.g. tacrine, donepezil and rivastigmine that have been reported to cause gastrointestinal disturbances and problems associated with bioavailability. Therefore, the search for new AChE inhibitors with higher efficacy, particularly from natural products, should be an important issue for search.

In vitro tests for screening antimicrobial activity were performed using Gram-positive bacteria ( $S$. aureus and E. faecalis), Gram-negative bacteria (P. aeruginosa and $E$. coli) and yeasts (C. albicans and $S$. cerevisiae). The antimicrobial activity assay was carried out by the preliminary well diffusion method. Only $P$. ramosior acetonic extract showed an inhibition growth against $S$. aureus and C. albicans, with no significant inhibition against Gram-negative bacteria. This results were in agreement with previous results for other Plectranthus spp. which also did not show antimicrobial activity against Gram negative bacteria ${ }^{(14)}$.

There has been a growing interest to find natural antioxidants, including volatile chemicals, in plants due to their inhibiting oxidative damage ${ }^{(3)}$. The acetonic extracts under study showed low antioxidant activity. In previous studies on Plectranthus spp. extracts, the high content in rosmarinic acid was associated to high antioxidant activity and high $\mathrm{AChE}$ inhibition, on aque-

\section{Discussão}

No presente estudo, foi avaliada a inibição da acetilcolinesterase, as atividades antioxidantes e antimicrobianas e a toxicidade no modelo Artemia salina de sete espécies de Plectranthus (P. swynnertonii, P. welwischii, $P$. woodii, $P$. cylindraceus, $P$. spicatus, $P$. ramosior e $P$. petiolaris). Os extratos acetónicos das sete espécies de Plectranthus foram preparados utilizando um método de extração assistido por ultrassons. Os rendimentos de extração foram determinados para todos os extratos preparados, e o extrato acetónico de $P$. ramosior revelou maiores rendimentos (Figura 1). Assim, o rendimento de extração de $P$. ramosior está relacionado com a maior extensão da ruptura celular do material vegetal. Isto é provavelmente devido à extração de ultrassons que causa a migração de compostos a partir do material vegetal, proporcionando um extrato enriquecido em comparação com os outros extratos em estudo ${ }^{(20)}$.

Em relação à inibição da $\mathrm{AChE}$ in vitro, o extrato acetónico de $P$. cylindraceus mostrou a maior inibição (Figura 2) e pode estar relacionado com o maior teor de ácido rosmarínico, uma vez que foi relatada uma correlação entre o conteúdo de ácido rosmarínico e a capacidade de inibição da AChE em quatro outras espécies de Plectranthus (P. ecklonii, P. fruticosus, P. lanuginosus e P. verticillatus) ${ }^{(20)}$. A inibição da AChE destas espécies de Plectranthus em estudo foi relativamente baixa em comparação com alguns dos medicamentos sintéticos utilizados, como a tacrina, donepezilo e rivastigmina, que foram relatados como causadores de distúrbios gastrointestinais e problemas associados à sua biodisponibilidade. Portanto, a busca por novos inibidores da AChE, particularmente a partir de produtos naturais, com maior eficácia deve ser um assunto importante a pesquisar.

Realizaram-se testes in vitro para rastreio da atividade antimicrobiana utilizando bactérias Gram-positivas $(S$. aureus e E. faecalis), bactérias Gram-negativas ( $P$. aeruginosa e E. coli) e leveduras (C. albicans e $S$. cerevisiae). $\mathrm{Na}$ atividade antimicrobiana realizada pelo método preliminar de difusão em poços, apenas o extrato $P$. ramosior mostrou uma inibição do crescimento contra $S$. aureus e C. albicans, e não mostrou uma inibição significativa contra bactérias Gram-negativas. Estes resultados estão de acordo com resultados anteriores para outras plantas do género Plectranthus que também não mostraram atividade antimicrobiana contra bactérias Gram-negativas ${ }^{(12)}$.

Tem havido um interesse crescente para encontrar antioxidantes naturais, incluindo produtos químicos voláteis, nas plantas devido à inibição do dano oxidativo 
ous extracts ${ }^{(20)}$. Since the acetonic extracts in study showed moderate AChE inhibition and also moderate antioxidant activity, the rosmarinic acid content should be low, as well as other antioxidant extract constituents. Although these results showed that $P$. ramosior was the most antioxidant, the activity was only moderate in comparison with the controls, and, additionally its $\mathrm{AChE} \%$ inhibition was low. Hence this extract is not a potential starting point for the search of drugs that inhibit oxidative damage and may consequently prevent inflammatory conditions, ageing and neurodegenerative diseases (such as Alzheimer's disease) ${ }^{(18)}$.

In this study, the brine shrimp lethality assay (Artemia salina) was preliminary used to search for the toxicity of all extracts prepared. Only three extracts were considered toxic to A. salina (lethality $>40 \%$ ), and for those the LC50 was evaluated. P. swynnertonnii extract was the most toxic with LC50 value of $0.036 \mathrm{mg} / \mathrm{L}$, followed by $P$. cylindraceaus (LC50 $0.50 \mathrm{mg} / \mathrm{L}$ ) and $P$. ramosior (LC50 value $0.88 \mathrm{mg} / \mathrm{L}$ ). P. cylindraceaus extract showed the highest AChE \% inhibition and was also toxic to the Artemia model. Hence, this extract is an interesting starting point for future phytochemical studies to identify which compounds are responsible for the biological activities shown.

P. ramosior acetonic extract seemed to be the most promising extract regarding its activity: it had effect on growth inhibition against bacteria and yeast, had antioxidant activity and revealed high toxicity to A. salina model. However, the antimicrobial activity might be due to its toxicity and this should be further investigated. Other bioactivity studies should be carried out in order to unravel other potential bioactivities and its bioactive compounds.

Consequently, this bioactivity screening of Plectranthus extracts is important as a preliminary study to select which plants should be investigated for further phytochemical studies. Moreover, future studies on $P$. ramosior and other active extracts (such as $P$. cylindraceaus, $P$. swynnertonnii and $P$. petiolaris) could be done to determine which are the bioactive compounds and their structural elucidation (by spectroscopic methods), and confirm the bioactivity of these compounds that are responsible for the biological activity revealed in this study.

\section{Conclusion}

In the present study, seven species of Plectranthus were analysed to evaluate their acetylcholinesterase inhibition, antimicrobial and antioxidant activities and toxicity. None of the extracts showed high acetylcholinester-
(3). Os extratos acetónicos estudados mostraram baixa atividade antioxidante. Em estudos anteriores sobre extratos de Plectranthus, o alto teor de ácido rosmarínico foi associado a alta atividade antioxidante e alta inibição da AChE, em extratos aquosos ${ }^{(20)}$. Uma vez que os extratos de acetona no estudo mostraram uma inibição moderada da AChE bem como a atividade antioxidante, o teor de ácido rosmarínico deve ser baixo. Embora estes resultados tenham mostrado que $P$. ramosior foi o mais antioxidante, a atividade foi apenas moderada em comparação com os controlos e, adicionalmente, a sua inibição de AChE foi baixa. Por conseguinte, este extrato não é um potencial ponto de partida para a pesquisa de fármacos que inibem o dano oxidativo, podendo consequentemente prevenir condições inflamatórias, envelhecimento e doenças neurodegenerativas (como a doença de Alzheimer) ${ }^{(18)}$.

O ensaio de letalidade no modelo da Artemia salina, foi utilizado preliminarmente para pesquisar a toxicidade de todos os extratos preparados neste estudo. Apenas três extratos foram considerados tóxicos para A. sali$n a$ (letalidade $>40 \%$ ), e para os que foram avaliados a $\mathrm{LC}_{50}$. O extrato de P. swynnertonnii foi o mais tóxico com $\mathrm{LC}_{50}$ de $0,036 \mathrm{mg} / \mathrm{L}$, seguido do $P$. cylindraceaus $\left(\mathrm{LC}_{50}-0,50 \mathrm{mg} / \mathrm{L}\right)$ e $P$. ramosior $\left(\mathrm{LC}_{50}-0,88 \mathrm{mg} / \mathrm{L}\right) . \mathrm{O}$ extrato de $P$. cylindraceaus apresentou a maior inibição de AChE e também foi tóxico para o modelo de Artemia. Assim, este extrato é um ponto de partida interessante para futuros estudos fitoquímicos, de modo a poder identificar quais os compostos que são responsáveis pelas atividades biológicas mostradas.

O extrato acetónico de $P$. ramosior parece ser o extrato mais promissor em relação às atividades biológicas estudadas: obteve efeito sobre a inibição do crescimento contra bactérias e leveduras em estudo, obteve atividade antioxidante e revelou alta toxicidade ao modelo de A. salina. No entanto, a actividade antimicrobiana pode ser devida à sua toxicidade e isto deve ser investigado mais a fundo. Outros estudos de bioactividade devem ser realizados para desvendar outras potenciais bioactividades e quais os compostos bioactivos responsáveis pelas actividades reveladas.

Consequentemente, este rastreio de bioactividade de extractos de Plectranthus é importante como um estudo preliminar, para selecionar quais as plantas que devem ser investigadas para estudos fitoquímicos adicionais. Além disso, estudos futuros sobre $P$. ramosior e outros extratos ativos (como P. cylindraceaus, P. swynnertonnii e $P$. petiolaris) podem ser realizados para revelar quais são os compostos bioativos e sua elucidação estrutural (por métodos espectroscópicos) e confirmar a bioatividade desses compostos que são responsáveis pela atividade biológica revelada. 
ase inhibition or antioxidant activities. Only P. ramosior acetonic extract exhibited antimicrobial activity, showing positive results against the Gram-positive bacteria and yeasts tested. Three extracts were toxic against the brine shrimp, with $P$. swynnertonnii being the most toxic. P. ramosior acetonic extract exhibited antimicrobial activity. This preliminary study allows the selection of which plants should be chosen for future studies. Further studies should be undertaken to identify the bioactive compounds responsible for these bioactivities of the active extracts. ( $P$. ramosior, $P$. swynnertonnii, $P$. cylindraceaus and $P$. petiolaris).

\section{Conflict of interest}

The authors declares that there are no financial or personal relationships that could be viewed as a potential conflict of interest.

\section{Conclusão}

No presente estudo, sete espécies de Plectranthus foram analisadas para avaliar a inibição da acetilcolinesterase, atividades antimicrobiana e antioxidante, e toxicidade. Nenhum dos extratos mostrou elevada inibição de acetilcolinesterase ou atividade antioxidante. Somente o extrato acetónico de $P$. ramosior mostrou resultados positivos contra bactérias Gram-positivas e leveduras testadas. Três extratos foram tóxicos contra o modelo de $A$. salina, sendo P. swynnertonnii o mais tóxico. O extrato acetónico de $P$. ramosior exibiu actividade antimicrobiana. Este estudo preliminar permite selecionar quais as plantas que devem ser escolhidas em estudos futuros. Outros estudos devem ser realizados para identificar os compostos bioativos responsáveis por essas bioatividades dos extratos ativos (P. ramosior, P. swynnertonnii, $P$. cylindraceaus e $P$. petiolaris).

\section{Conflito de Interesses}

Os autores declaram não existir qualquer relação de natureza financeira ou pessoal que possa ser entendida ou representar um potencial conflito de interesses. 


\section{References/Referências}

1. Lukhoba CW, Simmonds MSJ, Paton AJ. Plectranthus: A review of ethnobotanical uses. J Ethnopharmacol. 2006;103(1):1-24.

2. Rice LJ, Brits GJ, Potgieter CJ, Van Staden J. Plectranthus: A plant for the future? South African J Bot. 2011;77(4):947-59.

3. Rijo P, Batista M, Matos M, Rocha E, Jesus S, Simões M. Screening of antioxidant and antimicrobial activities on Plectranthus spp. extracts. Biomed Biopharm Res. 2012;9(2):225-35.

4. Rijo P, Matias D, Fernandes AS, Simões MF, Nicolai M, Reis CP. Antimicrobial plant extracts encapsulated into polymeric beads for potential application on the skin. Polymers (Basel). 2014;6(2):479-90.

5. Davinelli S, Sapere N, Zella D, Bracale R, Intrieri M, Scapagnini G. Pleiotropic Protective Effects of Phytochemicals in Alzheimer's Disease. Oxid Med Cell Longev. 2012;2012:1-11.

6. Tabet N. Acetylcholinesterase inhibitors for Alzheimer's disease: anti-inflammatories in acetylcholine clothing! Age Ageing. 2006;35(4):336-8.

7. Colović MB, Krstić DZ, Lazarević-Pašti TD, Bondžić AM, Vasić VM. Acetylcholinesterase inhibitors: pharmacology and toxicology. Curr Neuropharmacol. 2013;11(3):315-35.

8. Sung SH, Kang SY, Lee KY, Park MJ, Kim JH, Park JH, et al. (+)-Alpha-viniferin, a stilbene trimer from Caragana chamlague, inhibits acetylcholinesterase. Biol Pharm Bull. 2002;25(1):125-7.

9. Duracková Z. Some current insights into oxidative stress. Physiol Res. 2010;59(4):459-69.

10. Bonomini F, Rodella LF, Rezzani R. Metabolic syndrome, aging and involvement of oxidative stress. Aging Dis. 2015;6(2):109-20.

11. Guimarães DO, Momesso L da S, Pupo MT. Antibióticos: importância terapêutica e perspectivas para a descoberta e desenvolvimento de novos agentes. Quim Nova. 2010;33(3):667-79.

12. Kubínová R, Pořízková R, Navrátilová A, Farsa O, Hanáková Z, Bačinská A, et al. Antimicrobial and enzyme inhibitory activities of the constituents of Plectranthus madagascariensis (Pers.) Benth. J Enzyme Inhib Med Chem. 2014;29(5):749-52.

13. Albar H. Phytochemical composition and antimicrobial activities of the essential oil from Plectranthus tenuiflorus growing in Saudi Arabia. KauEduSa. $2002 ; 1-9$

14. Rijo P, Batista M, Matos M, Rocha H, Jesus S, Simões MF. Screening of antioxidant and antimicrobial activities on Plectranthus spp. extracts. Biomed Biopharm Res. 2012;9(2):225-35.

15. Karchesy YM, Kelsey RG, Constantine G, Karchesy JJ. Biological screening of selected Pacific Northwest forest plants using the brine shrimp (Artemia salina) toxicity bioassay. Springerplus. 2016;5:510-8.

16. Chen X, Guo C, Kong J. Oxidative stress in neurodegenerative diseases. Neural Regen Res. 2012;7(5):376-85.

17. Hamidi MR, Jovanova B, Panovska TK. Toxicological evaluation of the plant products using Brine Shrimp (Artemia salina L.) model. Maced Pharm Bull. 2014;60(1):9-18

18. Alanís-Garza BA, González-González GM, Salazar-Aranda R, Waksman de Torres N, Rivas-Galindo VM. Screening of antifungal activity of plants from the northeast of Mexico. J Ethnopharmacol. 2007;114(3):468-71.

19. Meyer B, Ferrigni N, Putnam J, Jacobsen L, Nichols D, McLaughlin J. Brine Shrimp: A Convenient General Bioassay for Active Plant Constituents. Planta Med. 1982;45(5):31-4.

20. Rijo P, Falé PL, Serralheiro ML, Simões MF, Gomes A, Reis C. Optimization of medicinal plant extraction methods and their encapsulation through extrusion technology. Measurement. 2014;58:249-55. 